\title{
Anticancer activity of 23,24-dihydrocucurbitacin B against the HeLa human cervical cell line is due to apoptosis and $\mathrm{G}_{2} / \mathrm{M}$ cell cycle arrest
}

\author{
JUN-XIAO ZHANG $^{1 *}$, HONG WEI-TAN ${ }^{1 *}$, CHUN-YAN HU ${ }^{1}$, WEI-QIANG WANG ${ }^{1}$, \\ GUANG-HUA CHU ${ }^{1}$, LI-HUI WEI ${ }^{2}$ and LIU CHEN $^{1}$ \\ ${ }^{1}$ Department of Gynecology, Northwest Women and Children's Hospital, Xi'an, Shaanxi 710061; \\ ${ }^{2}$ Department of Gynecology, Peking University People's Hospital, Beijing 100044, P.R. China
}

Received August 29, 2017; Accepted November 17, 2017

DOI: $10.3892 /$ etm.2018.5710

\begin{abstract}
Cervical cancer is one of the primary causes of cancer-associated mortality worldwide. Due to the increasing incidence of cervical cancer, multiple treatment options are required. Initial responses to chemotherapy and surgical interventions are generally positive, however patients often experience relapse and tumor recurrence. Currently, the effects of cucurbitacins on different types of cancer are being investigated, as they exhibit a wide variety of bioactivities. The anticancer activity of the cucurbitacin 23,24-dihydrocucurbitacin B against a panel of human cervical cancer cell lines was investigated in the current study. Cell viability was determined using an MTT assay and apoptosis was detected using DAPI staining. The proportion of apoptotic cells, cell cycle distribution, mitochondrial membrane potential $\left(\Delta \Psi_{\mathrm{m}}\right)$ and reactive oxygen species (ROS) levels were estimated using flow cytometry. Protein expression was determined using western blot analysis. The results of the current study indicated that 23,24-dihydrocucurbitacin B inhibited the viability of human cervical cancer cell lines and had an $\mathrm{IC}_{50}$ of 40-60 $\mu \mathrm{M}$. However, its cytotoxic effects were much less pronounced in normal epithelial fr2 and HerEpiC cells, where it had an $\mathrm{IC}_{50}$ of $125 \mu \mathrm{M}$. The underlying mechanisms of this were further studied and the results demonstrated that 23,24-dihydrocucurbitacin B induced apoptosis in HeLa cells and caused ROS-mediated shifts in the $\Delta \Psi_{\mathrm{m}}$. Additionally, it caused the cell cycle arrest of HeLa cells at the $G_{2} / M$ checkpoint. The phosphoinositide 3 kinase/protein kinase
\end{abstract}

Correspondence to: Dr Liu Chen, Department of Gynecology, Northwest Women and Children's Hospital, 106 Yan Xiang Road, Xi'an, Shaanxi 710061, P.R China

E-mail: liuchen7x@gmail.com

"Contributed equally

Key words: cervical carcinoma, mechanistic target of rampamycin, reactive oxygen species, 23,24-dihydrocucurbitacin B
$\mathrm{B} / \mathrm{mechanistic} \mathrm{target} \mathrm{of} \mathrm{rampamycin} \mathrm{(PI3K/AKT/mTOR)}$ cascade may serve an important role in cancer tumorigenesis, progression and resistance to chemotherapy. The results indicated that 23,24-dihydrocucurbitacin B significantly decreased the expression of important proteins in the $\mathrm{PI} 3 \mathrm{~K} / \mathrm{Akt} / \mathrm{mTOR}$ cascade. Taken together, these results suggest that 23,24-dihydrocucurbitacin B may be novel method of treating cervical cancer.

\section{Introduction}

Cucurbitacins are secondary plant metabolites chemically categorized as steroids. They are synthesized by a range of plant species, particularly by those that are part of the cucurbitaceae family (1). Cucurbitacins normally exist as glycosides and help plants to deter predators (1). It has been reported that cucurbitacins exhibit anticancer activities against different types of cancer (2-4); however, the antitumor activity of 23,24-cucurbitacin B against cervical cancer cells remains unclear. Therefore, the present study aimed to investigate the anticancer effects of 23,24-dihydrocucurbitacin B against the human HeLa cervical cancer cell line. Cervical cancer is the third most commonly diagnosed cancer in females worldwide. Every year, >500,000 females are diagnosed with cervical cancer, which accounts for $\sim 9 \%$ of all newly diagnosed cancer cases globally (5). Existing treatments, including radical hysterectomy and radiotherapy, have outcomes; however, cervical cancer continues to account for $\sim 300,000$ mortalities every year (5). Surgery is the only appropriate option for early stage cervical cancer and the majority of cervical cancers are diagnosed at advanced stages (5). Advanced stage cervical cancers are treated with radiotherapy, which induces severe side effects, including skin reactions, hair loss, pain, tiredness and fatigue and lymphodeama, which affect the patient's quality of life (6).

The present study evaluated the effect of the cucurbitacin 23,24-dihydrocucurbitacin B on human cervical cancer cells. Its underlying mechanism of action was assessed with particular emphasis on the effect of 23,24-dihydrocucurbitacin B on the phosphoinositide 3 kinase/protein kinase B/mechanistic target of rampamycin (PI3K/Akt/mTOR) cascade. The 
expression of proteins in the $\mathrm{PI} 3 \mathrm{~K} / \mathrm{Akt} / \mathrm{mTOR}$ pathway is dysregulated in several types of cancer (7). The first generation of molecules, including rapamycin and its analogues that inhibit mTOR also exhibit potent anticancer activity against different types of cancer, including pancreatic, cervical, ovarian and breast cancer (7). Currently, P13K, Akt and the second generation of molecules, including temsirolimus, everolimus, and deforolimus that inhibit mTOR are being investigated in clinical trials $(7,8)$. The current study determined the effect of 23,24-dihydrocucurbitacin B on apoptosis, reactive oxygen species (ROS) levels, the mitochondrial membrane potential $\left(\Delta \Psi_{\mathrm{m}}\right)$ and the cell cycle of cells from the human cervical HeLa cancer cell line. Additionally, the effect of 23,24-dihydrocucurbitacin B on the expression of important proteins within the PI3K/Akt/mTOR signaling pathway was evaluated. The aim of the current study was to identify whether 23,24-dihydrocucurbitacin B exhibits significant anticancer activity, in order to determine whether 23,24-dihydrocucurbitacin B may be developed as a novel method of treating cervical cancer.

\section{Materials and methods}

Cell culture conditions. The cervical cancer cell lines C33A, ME-180, C4-1 and HeLa, the normal cell line fR2 and human cervical epithelial cells (HCerEpiC) were obtained from the Cancer Research Institute of Beijing (Beijing, China) and maintained in Dulbecco's modified Eagle's medium (Invitrogen; Thermo Fisher Scientific, Inc., Waltham, MA, USA) supplemented with $10 \%$ fetal bovine serum (Invitrogen; Thermo Fisher Scientific, Inc.), $100 \mu \mathrm{g} / \mathrm{ml}$ streptomycin and $100 \mathrm{U} / \mathrm{ml}$ penicillin G (HiMedia, West Chester, Pennsylvania, USA) in an incubator at $37^{\circ} \mathrm{C}$ with $5 \% \mathrm{CO}_{2}$.

MTT assay. The effect of 23,24-dihydrocucurbitacin B (Sigma-Aldrich; Merck KGaA, Darmstadt, Germany) on the viability of different cervical cancer cell lines and normal fR2 and HCerEpiC cells was evaluated using an MTT assay. Cells were seeded at $1 \times 10^{6}$ cells/well in 96-well plates for $12 \mathrm{~h}$ and then treated with different concentrations of 23,24-dihydrocucurbitacin B $(0,0.78,1.56,3.12,6.25,12.5$, $25,50,100$ and $200 \mu \mathrm{M}$ ) for $24 \mathrm{~h}$. A total of $20 \mu \mathrm{l}$ MTT solution $(2.5 \mathrm{mg} / \mathrm{ml})$ for $24 \mathrm{~h}$ was then added to each well. The medium was removed and $500 \mu 1$ dimethyl sulfoxide was added to each well to dissolve formazan crystals. Optical density was recorded using an ELISA plate reader at a wavelength of $570 \mathrm{~nm}$. 23,24-dihydrocucurbitacin B exhibited marked anticancer activity against all cell lines; however, further experiments were performed on the HeLa cancer cell line alone as the lowest MIC was observed against this cell line.

Colony formation assay. HeLa cells were cultured to the exponential phase (70\% confluence), collected and counted using a hemocytometer. Cells were then seeded at a density of 200 cells/well and incubated for $24 \mathrm{~h}$ to allow cells to adhere. Cells were then treated with different concentrations of 23,24-dihydrocucurbitacin B $(0,20,40$ and $80 \mu \mathrm{M})$. Cells were incubated for 6 days and then washed with PBS. This
Table I. IC $_{50}$ of 23,24-dihydrocucurbitacin B against different cervical cancer and normal cell lines as determined by MTT assay.

\begin{tabular}{lc}
\hline Cell line & $\mathrm{IC}_{50}(\mu \mathrm{M})$ \\
\hline C33A & 60 \\
ME-180 & 50 \\
C4-1 & 40 \\
HeLa & 40 \\
FR2 & 125 \\
HCerEpiC & 125 \\
\hline
\end{tabular}

$\mathrm{IC}_{50}$, half maximal inhibitory concentration.

was followed by fixation with $70 \%$ methanol at $-20^{\circ} \mathrm{C}$ for $24 \mathrm{~h}$ and staining with $0.01 \%$ (w/v) crystal violet for $35 \mathrm{~min}$ at $25^{\circ} \mathrm{C}$. Cells were then counted in 10 fields using a light microscope at a magnification of $\times 200$.

Apoptosis detection. HeLa cells were cultured to a density of $2 \times 10^{5}$ cells/well in 6 -well plates and were subsequently treated with $0,20,40$ and $80 \mu \mathrm{M}$ 23,24-dihydrocucurbitacin B for $24 \mathrm{~h}$. Cells were then stained with DAPI for $20 \mathrm{~min}$ at room temperature. The cells were then fixed with $70 \%$ methanol at $-20^{\circ} \mathrm{C}$ overnight and observed using fluorescence microscopy (magnification, x200). A similar procedure was followed for Annexin V-fluorescein isothiocyanate (FITC)/propidium iodide (PI) (Sigma-Aldrich; Merck KGaA) staining; cells were stained with annexin V/PI and investigated using a flow cytometer, (BD Biosciences, San Jose, CA, USA) following the manufacturer's protocol and BD FACSuite software version 1.0 for analysis.

Estimation of ROS and $\Delta \Psi_{m}$. HeLa cells were seeded at a density of $2 \times 10^{5}$ cells/well in 6 -well plates and incubated for $24 \mathrm{~h}$. Cells were then treated with $0,20,40$ and $80 \mu \mathrm{M}$ 23,24-dihydrocucurbitacin $\mathrm{B}$ for $24 \mathrm{~h}$ at $37^{\circ} \mathrm{C}$ in $5 \% \mathrm{CO}_{2}$. Cells were washed twice with $\mathrm{PBS}$ and resuspended in $500 \mu 1$ dihydrofluorescein diacetate $(10 \mu \mathrm{M})$ (Sigma-Aldrich; Merck KGaA) for mitochondrial ROS estimation and $\mathrm{DiOC}_{6}(1 \mu \mathrm{mol} / \mathrm{l})$ at $37^{\circ} \mathrm{C}$ in a dark room for $35 \mathrm{~min}$ to measure the $\Delta \Psi_{\mathrm{m}}$. Samples were then investigated using a flow cytometer following a previously described protocol $(9,10)$.

Cell cycle distribution of HeLa cells using flow cytometry. HeLa cells were harvested and washed twice with PBS. Cells were then fixed with $70 \%$ ethanol for $\sim 1 \mathrm{~h}$ at $-20^{\circ} \mathrm{C}$ and then washed again with PBS. Cells were resuspended in a solution of PI (50 $\mu \mathrm{l} / \mathrm{ml})$ and RNasel $(250 \mu \mathrm{g} / \mathrm{ml})$ (Invitrogen; Thermo Fisher Scientific, Inc.). This was followed by incubation for $30 \mathrm{~min}$ at room temperature and fluorescence-activated cell sorting using 10,000 cells/group with a flow cytometer.

Western blot analysis. Following treatment with various concentrations of 23,24-dihydrocucurbitacin B, cells were harvested and lysed in radioimmunoprecipitation lysis buffer (20 mM HEPES, $350 \mathrm{mM} \mathrm{NaCl,} \mathrm{20 \%} \mathrm{glycerol,} \mathrm{1 \%}$ 


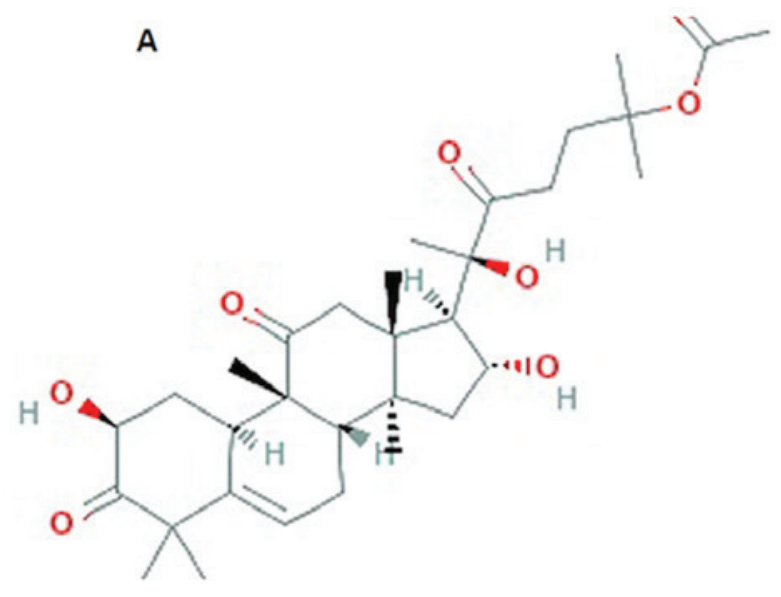

B
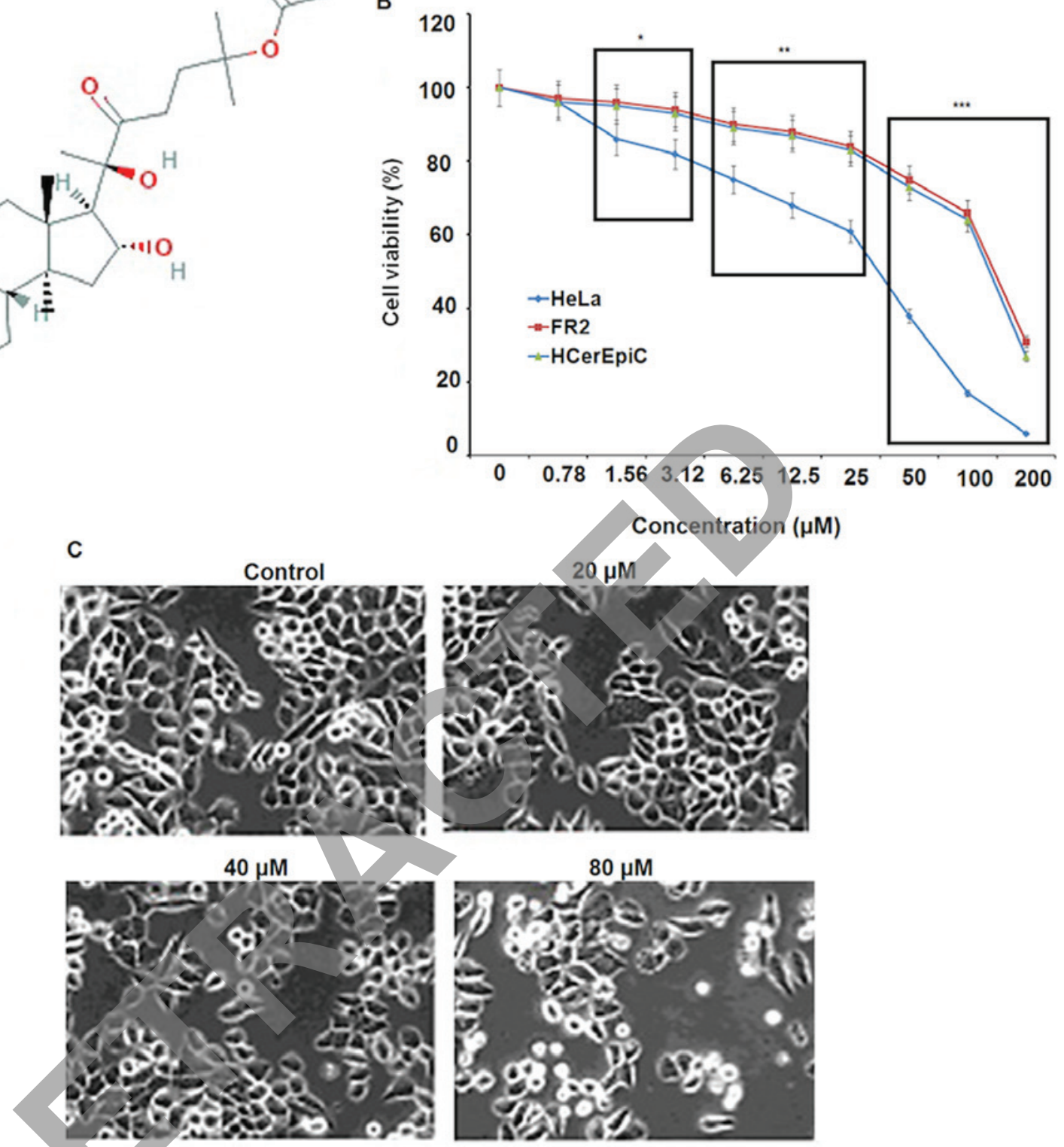

Figure 1. The effect of 23,24-dihydrocucurbitacin B on the HeLa cervical cancer cell line. (A) Chemical structure of 23,24-dihydrocucurbitacin B. (B) Effect of 0,20, 40 and $80 \mu \mathrm{M} 23,24$-dihydrocucurbitacin B on the viability of HeLa cervical cancer cells. (C) Effect of 0, 20, 40 and 80 $\mu \mathrm{M} 23,24$-dihydrocucurbitacin B on the morphology of HeLa cervical cancer cells. Magnification, x200. All experiments were performed in triplicate and values are expressed as the mean \pm standard deviation. The differences between the two cell lines at indicated concentrations were considered significant at ${ }^{*} \mathrm{P}<0.01,{ }^{* *} \mathrm{P}<0.001$ and ${ }_{* * * *} \mathrm{P}<0.0001$ at different doses between the two cell lines (HeLa vs. HCerEPiC).

Nonidet P 40, $1 \mathrm{mM} \mathrm{MgCl} 2,0.5 \mathrm{mM}$ EDTA, 0.1 mM EGTA, $1 \mathrm{mM}$ dithiothreitol, $1 \mathrm{mM}$, phenylmethane sulfonyl fluoride, protease inhibitor cocktail and phosphatase inhibitor cocktail). The protein concentration was determined by BCA assay. A total of $20 \mu \mathrm{g}$ protein/lane was separated on $10 \%$ SDS-PAGE gel. Proteins were then transferred to nitrocellulose membranes, blocked with $5 \%$ bovine serum albumin (Invitrogen; Thermo Fisher Scientific,Inc.), for $45 \mathrm{~min}$ at room temperature and probed with the following primary antibodies overnight at $4^{\circ} \mathrm{C}$ : Actin (cat. no. sc-58673), Akt (cat. no. sc-135829), phosphorylated (p)-AKT (cat. no. sc-7985-R), P13K (cat. no. sc-136298), p-P13K (cat. no. sc-100407), mTOR (cat. no. sc-517464) and p-mTOR (cat. no. sc-293133; all 1:1,000). All antibodies were obtained from Santa Cruz Biotechnology, Inc. (Dallas, TX, USA). Proteins were then incubated with horseradish peroxidase-conjugated anti-rabbit secondary antibody (cat. no. sc-2357-CM) for $1 \mathrm{~h}$ overnight at $4^{\circ} \mathrm{C}$. WEST-SAVE Up ${ }^{\mathrm{TM}}$ luminal-based enhanced chemiluminescent reagent was then used to visualize bands (ABFrontier, Co., Ltd., Seoul, Korea).

Statistical analysis. Experiments were performed in triplicate and data are presented as the mean \pm standard deviation. Statistical analysis was performed by GraphPad prism 7 (GraphPad Software, Inc., La Jolla, CA, USA). Student's $t$ test was used for comparison between 2 samples and one way analysis of variance followed by a Tukey's post hoc test was used for comparisons between $>2$ samples. $\mathrm{P}<0.01$ was determined to indicate a statistically significant difference. 
A
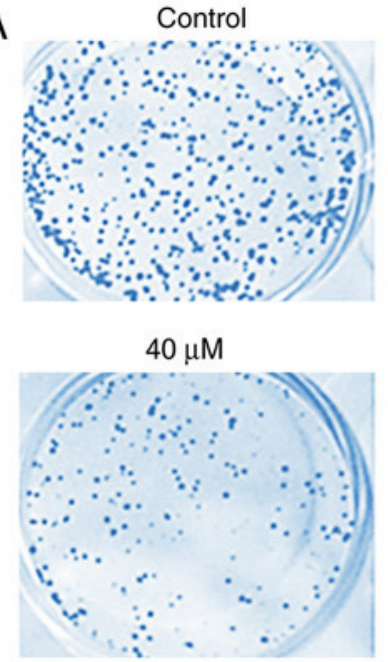
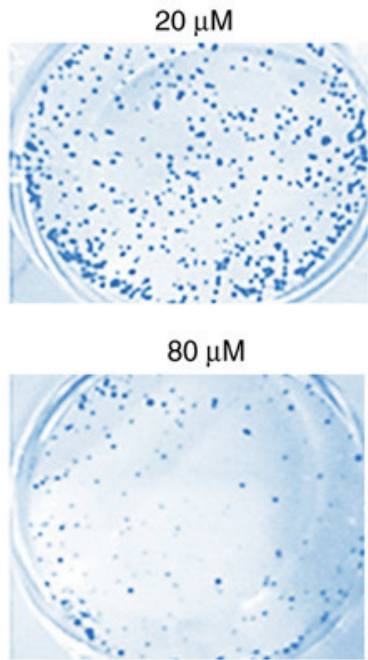

B

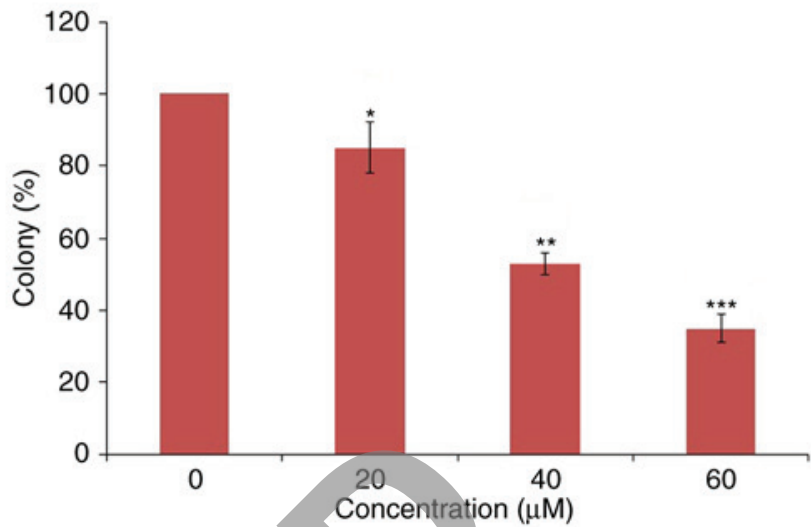

Figure 2. Effect of 23,24-dihydrocucurbitacin B on colony formation in HeLa cells. (A) Images of the colony formation potential of HeLa cells treated with 0, 20, 40 and $80 \mu \mathrm{M}$ 23,24-dihydrocucurbitacin B. (B) Quantification of colonies of HeLa cells at the indicated doses. All results are representative of three biological replicates and values are expressed as the mean \pm standard deviation. ${ }^{*} \mathrm{P}<0.01,{ }^{* *} \mathrm{P}<0.001$ and ${ }^{* * *} \mathrm{P}<0.0001$ vs. untreated cells.

\section{Results}

Cytotoxic potential of 23,24-dihydrocucurbitacin B on cervical cancer cells. The cytotoxic potential of 23,24-dihydrocucurbitacin B (Fig. 1A) was evaluated against a panel of human cervical cancer cell lines (Table I). The results indicated that 23,24-dihydrocucurbitacin B exhibits significant anticancer activity against all of the cervical cancer cell lines used in the present study. 23,24-dihydrocucurbitacin B exhibited dose-dependent activity with an $\mathrm{IC}_{50}$ of $40 \mu \mathrm{M}$ against HeLa cells (Fig. 1B). 23,24-dihydrocucurbitacin B exhibited significantly lower cytoxicity in fR-2 cells and HCerEpicCs $\left(\mathrm{IC}_{50}, 125 \mu \mathrm{M}\right)$ compared with HeLa cells. It also caused marked changes in the morphology of HeLa cells; cells exhibited shrinked membranes (Fig. 1C). A colony formation assay was performed and the results indicated that following 23,24-dihydrocucurbitacin B administration, the percentage of colonies of HeLa cells decreased in a dose-dependent manner (Fig. 2).

23,24-dihydrocucurbitacin B induces apoptosis in HeLa cells. DAPI staining was performed to investigate whether 23,24-dihydrocucurbitacin B exerts antiproliferative effects on HeLa cells by inducing apoptosis. The results of DAPI staining indicated that 23,24-dihydrocucurbitacin B caused marked apoptosis in HeLa cells (Fig. 3). Annexin V-FITC/PI staining and flow cytometry indicated that the percentage of apoptotic cells significantly increased after $24 \mathrm{~h}$ incubation with 20, 40 and $80 \mu \mathrm{M}$ 23,24-dihydrocucurbitacin $\mathrm{B}$, compared with untreated cells (Fig. 4). Higher concentrations of 23,24-dihydrocucurbitacin B induced a higher rate of apoptosis, indicating that 23,24-dihydrocucurbitacin B induces apoptosis in a concentration-dependent manner.

\section{3,24-dihydrocucurbitacin B causes ROS activation in HeLa} cells. The pro-apoptotic potential of 23,24-dihydrocucurbitacin B observed following DAPI staining suggested that it may cause the accumulation of intracellular ROS. Therefore
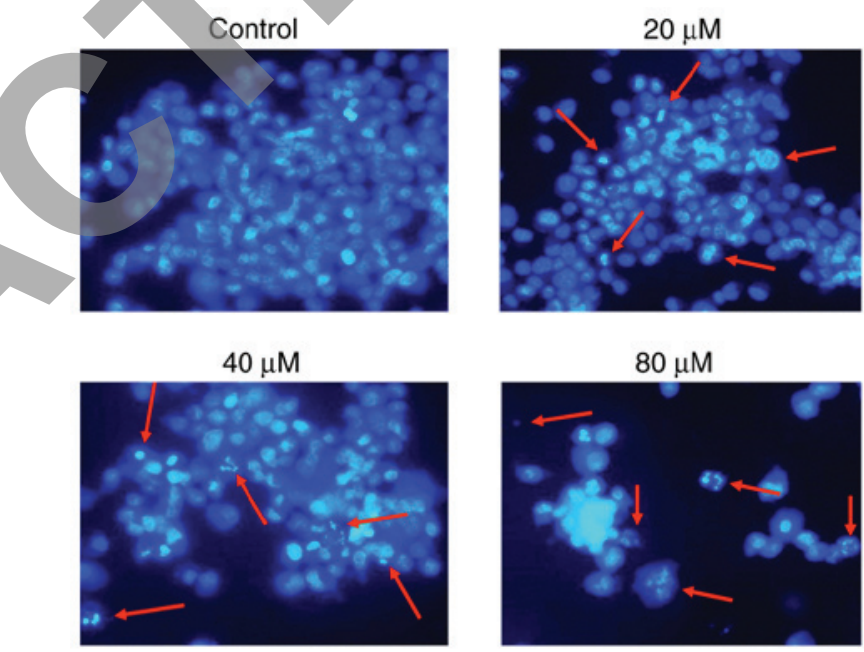

Figure 3. DAPI staining indicating the induction of apoptosis in HeLa cells treated with $0,20,40$ and $80 \mu \mathrm{M} 23,24$-dihydrocucurbitacin B. All results are representative of three biological experiments. Red arrows indicate apoptotic cells. Magnification, x200.

ROS levels were estimated in HeLa cells treated with different doses of 23,24-dihydrocucurbitacin B for $24 \mathrm{~h}$. The results indicated that the intracellular ROS levels of treated cells significantly increased, by $70-260 \%$ compared with untreated cells (Fig. 5A). This suggests that 23,24-dihydrocucurbitacin B serves an important role in stimulating the accumulation of ROS in HeLa cells, thereby inducing apoptosis.

23,24-dihydrocucurbitacin B lowers the $\Delta \Psi m$. ROS generation is associated with mitochondrial dysfunction, as it disturbs the outer mitochondrial potential in order to discharge apoptosis-promoting proteins (11). Therefore, varying concentrations of 23,24-dihydrocucurbitacin $\mathrm{B}$ were used to investigate its effect on the $\Delta \Psi_{\mathrm{m}}$ in HeLa cells. The results indicated that the $\Delta \Psi_{\mathrm{m}}$ of HeLa cells treated with 23,24-dihydrocucurbitacin B significantly decreased and that this decrease occurred in a dose-dependent manner (Fig. 5B). 


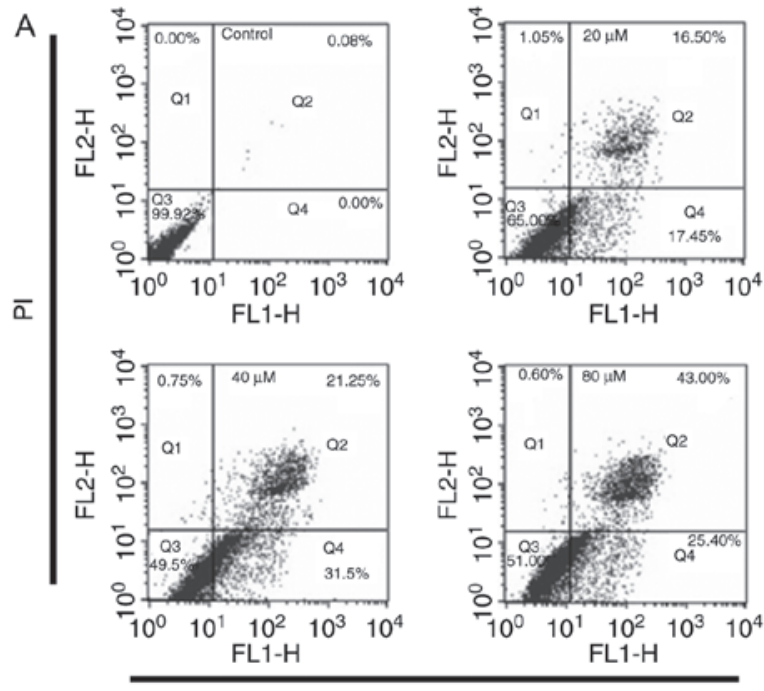

B

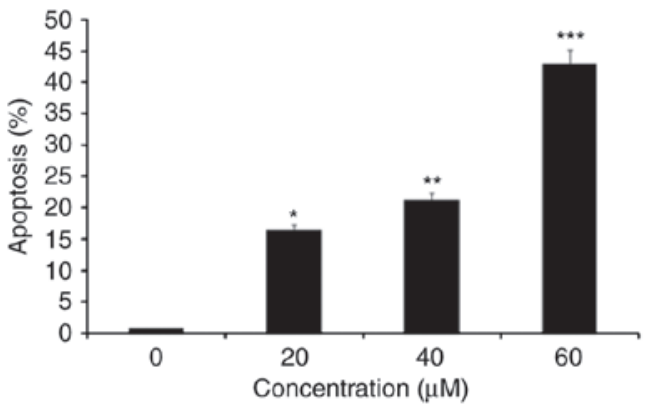

Annexin V-FITC

Figure 4. Estimation of apoptotic population of HeLa cells treated with 0, 20, 40 and $80 \mu \mathrm{M}$ 23,24-dihydrocucurbitacin B using Annexin V-FITC/PI staining and flow cytometry. (A) Flow cytometric analysis. (B) The apoptosis of HeLa cells. All results are representative of three biological experiments. All experiments were carried out in triplicate and values are expressed as the mean \pm standard deviation. ${ }^{*} \mathrm{P}<0.01,{ }^{* * *} \mathrm{P}<0.001$ and ${ }^{* * *} \mathrm{P}<0.0001$ vs. untreated cells. $\mathrm{PI}$, propidium iodide; FITC, fluorescein isothiocyanate.

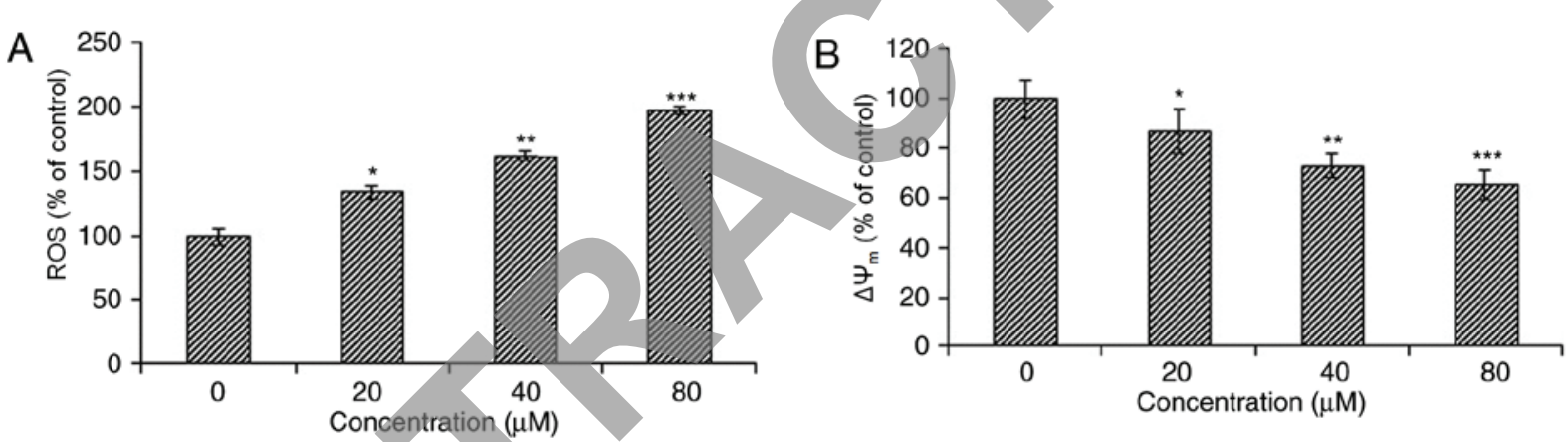

Figure 5. Effect of 0,20, 40 and $80 \mu \mathrm{M} 23,24$-dihydrocucurbitacin B on ROS generation and $\Delta \Psi m$. (A) ROS production. (B) $\Delta \Psi \mathrm{m}$. All experiments were performed in triplicate and values are expressed as the mean \pm standard deviation. ${ }^{*} \mathrm{P}<0.01,{ }^{* *} \mathrm{P}<0.001$ and ${ }^{* * *} \mathrm{P}<0.0001$ vs. untreated cells. ROS, reactive oxygen species; MMP, mitochondrial membrane potential.

23,24-dihydrocucurbitacin B induces cell cycle arrest. Cell cycle arrest is one of the important mechanisms by which anticancer agents exert their inhibitory effects (12). Therefore, the effect of 23,24-dihydrocucurbitacin B on the cell cycle of HeLa cancer cells was determined in the present study. The results indicated that the number of HeLa cells was markedly increased in the $\mathrm{G}_{2}$ phase of the cell cycle at doses of 20-80 $\mu \mathrm{M}$ of 23,24-dihydrocucurbitacin B, thereby inducing $\mathrm{G}_{2}$ arrest (Fig. 6). The proportion of HeLa cells in the $\mathrm{G}_{2}$ phase were slightly increased at a concentration of $20 \mu \mathrm{M}$, moderately increased at $40 \mu \mathrm{M}$ and highly increased at $80 \mu \mathrm{M}$ suggesting that 23,24-dihydrocucurbitacin $B$ induces the $\mathrm{G}_{2} / \mathrm{M}$ arrest of HeLa cancer cells in a dose-dependent manner.

23,24-dihydrocucurbitacin B targets the mTOR/PI3K/Akt signaling pathway. The effect of 23,24-dihydrocucurbitacin B on the expression of some of the proteins involved in the mTOR/PI3K/Akt cascade was evaluated using western blotting. The results indicated that the expression of mTOR, p-mTOR, PI3K, p-PI3K and p-Akt was markedly decreased in HeLa cells following treatment with 23,24-dihydrocucurbitacin B (Fig. 7). These decreases occurred in a dose-dependent manner.

\section{Discussion}

Cervical cancer is one of the most common types of cancer diagnosed in women worldwide and $\sim 5$ Lakh women are diagnosed with the disease annually (5). Existing treatment options, including radical hysterectomy and radiotherapy have good clinical outcomes; however, cervical cancer continues to account for a high number of cancer-associated mortalities. Surgery is the most appropriate treatment option if the cancer is detected at an early stage; other treatment options, such as radiotherapy have severe side effects, including skin reactions, hair loss, pain, tiredness and fatigue and lymphodeama, which adversely affect the patient's quality of life (6). Thus, the identification of novel treatments for cervical cancer that induce limited side effects is required. In the present study, the anticancer activity of 23,24-dihydrocucurbitacin B was evaluated in a panel of cervical cancer cell lines, including C33A, 


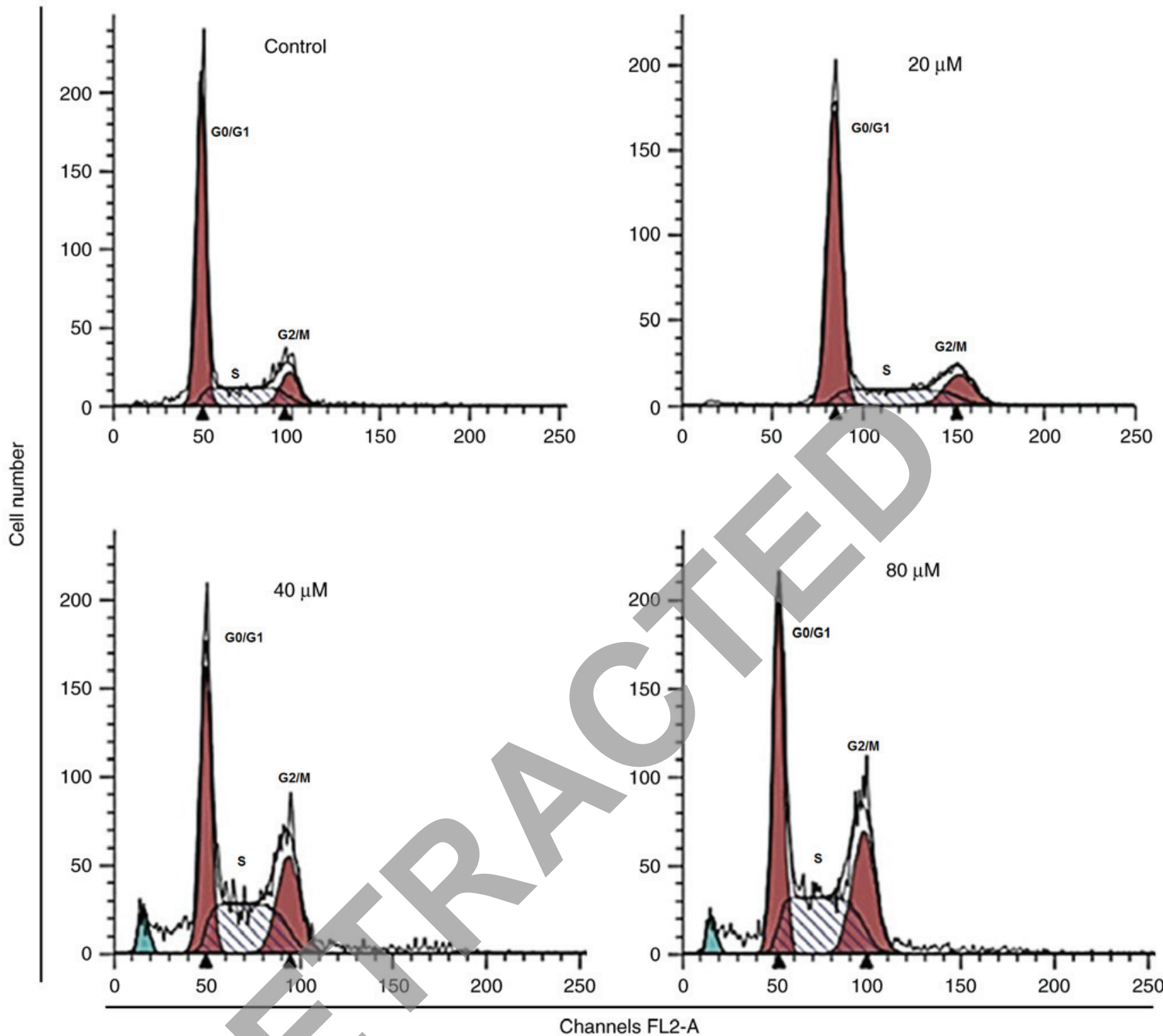

Figure 6. Effect of 0,20,40 and $80 \mu \mathrm{M} 23$,24-dihydrocucurbitacin B on the cell cycle distribution of HeLa cells. All results are representative of three biological experiments.

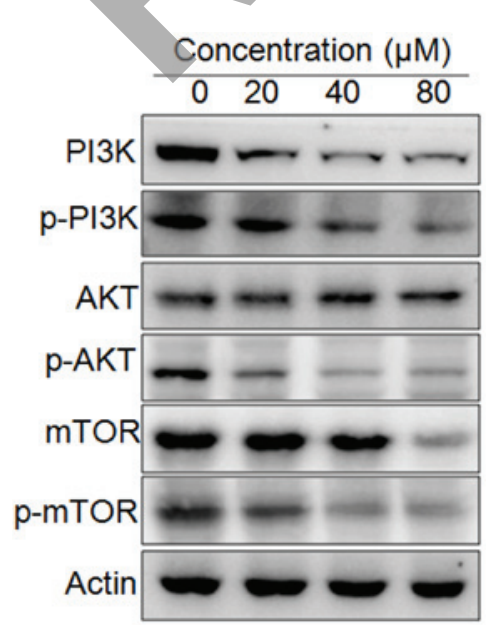

Figure 7. Effect of 0, 20, 40 and $80 \mu \mathrm{M} 23$,24-dihydrocucurbitacin $\mathrm{B}$ on the expression of mTOR/PI3K/Akt signaling pathway proteins in HeLa cells. All results are representative of three biological experiments. mTOR, mechanistic target of rampamycin; PI3K, phosphoinositol 3 kinase; Akt, protein kinase B; p-, phosphorylated.
ME-180, C4-1 and HeLa, and the normal cervical cell lines fR2 and HCerEpiC. 23,24-dihydrocucurbitacin B exhibited anticancer activity against all of the cervical cancer cell lines used. However, the highest activity was observed against HeLa and C4-1 cervical cancer cells. 23,24-dihydrocucurbitacin B exhibited an $\mathrm{IC}_{50}$ of $40 \mu \mathrm{M}$ against these two cell lines. The $\mathrm{IC}_{50}$ value of 23,24-dihydrocucurbitacin $\mathrm{B}$ was the same for the two cancer cell lines, however, only the HeLa cancer cell line was used for further experiments. Given these interesting results further studies should focus on evaluation of 23,24-dihydrocucurbitacin B against more cell lines. Although the $\mathrm{IC}_{50}$ of 23,24-dihydrocucurbitacin B is comparatively higher than that of known anticancer drugs, its low cytotoxicity against normal cervical cells suggests that it may be an effective anticancer molecule. However, 24-synthetic chemistry approaches may be required for the synthesis of more efficient derivatives (13). Furthermore, 23,24-dihydrocucurbitacin B exhibited low cytotoxicity in normal cells, indicating that it is selective for cancer cells. The results of the current study are 
consistent with those of previous studies, in which it was determined that 23,24-dihydrocucurbitacin B exhibits anticancer activity against a number of cancer cell types, including prostate and breast cancer cells $(13,14)$.

It has also been demonstrated that various anticancer drugs, including cisplatin, Taxon and 5-fluorouracil (12,15-19), exhibit anticancer effects by inducing apoptosis. Furthermore, the resistance of cancer cells to a particular drug is partially due to the resistance of cancer cells to apoptosis (20). In the current study, DAPI staining was performed to investigate whether 23,24-dihydrocucurbitacin B induces the apoptosis of HeLa cells. The results indicated that 23,24-dihydrocucurbitacin B induces apoptosis in a dose-dependent manner.

In addition, the results of the current study indicated that 23,24-dihydrocucurbitacin B-treated cells exhibited a reduction in the $\Delta \Psi \mathrm{m}$, which was mediated by ROS. These results are consistent with those of a previous study (18). Thus, the results of the present study indicate that 23,24-dihydrocucurbitacin B may induce apoptosis by increasing intracellular ROS and decreasing the $\Delta \Psi \mathrm{m}$. Several anticancer drugs act against cancer by producing $\operatorname{ROS}(21,22)$; for example, afferent A disrupts the $\Delta \Psi \mathrm{m}$ and induces oxidative stress, ultimately inducing apoptosis in osteosarcoma cells (23).

Flow cytometric analysis in the current study indicated that 23,24-dihydrocucurbitacin B induced $\mathrm{G}_{2} / \mathrm{M}$ cell cycle arrest and markedly increased the proportion of HeLa cells in the $\mathrm{G}_{2}$ phase in a dose-dependent manner. These results are consistent with those of a previous study, which demonstrated that 23,24-dihydrocucurbitacin $B$ induces $\mathrm{G}_{2} / \mathrm{M}$ cell cycle arrest in breast cancer cells (14). It is hypothesized that the $G_{2} / M$ cell cycle arrest of HeLa cells by 23,24-dihydrocucurbitacin B may be due to the regulation of cell cycle-associated proteins.

The PI3K/Akt/mTOR signaling pathway is considered to be an important target for anticancer chemotherapy (24). Therefore, the effect of 23,24-dihydrocucurbitacin B on the expression of important proteins, including mTOR, p-mTOR, $\mathrm{P} 13 \mathrm{~K}, \mathrm{p}-\mathrm{PI} 3 \mathrm{~K}, \mathrm{Akt}$ and $\mathrm{p}-\mathrm{Akt}$, were studied using western blotting. The results indicated that 23,24-dihydrocucurbitacin B-administrated cells exhibited a dose-dependent down regulation of $\mathrm{mTOR}$ and $\mathrm{p}$-mTOR proteins. There was also a decrease in the expression of PI3K and p-Akt in HeLa cells treated with 23,24-dihydrocucurbitacin B. The inhibition of the PI3K/AKT/mTOR pathway may be due to a decrease in the expression of mTOR and PI3K or the inhibition of their phosphorylation (25). However, further studies are required in order to confirm this. The PI3K/AKT/mTOR pathway serves a role in a number of cellular processes, including the proliferation and survival in several cell types and dysregulation of the P13K/AKT pathway is considered to be an important step in the pathogenesis of many diseases, including cancer $(25,26)$. Furthermore, dysregulated mTOR stimulation has also been reported to serve a key part in the development of nephropathy and the pathogenesis of HIV-associated malignancies (27). Therefore, the role of mTOR in the pathogenesis of HIV-associated disorders and cancer suggests that the use of specific P13K/AKT/mTOR inhibitors may be a novel approach to prevent and treat these diseases $(27,28)$. 23,24-dihydrocucurbitacin B inhibits this pathway; therefore, it may be effective at preventing the progression of diseases in which mTOR is upregulated. It should be noted that in the current study, the HeLa cell line was the only cell line used in subsequent experiments following preliminary screening of 23,24-dihydrocucurbitacin B against a panel of cervical cancer cell lines. Therefore, further studies focusing on the effect of 23,24-dihydrocucurbitacin B against other cell lines and subsequent in vivo studies are required to provide broader insights into the underlying mechanisms of 23,24-dihydrocucurbitacin B.

In conclusion, the results of the current study indicate that 23,24-dihydrocucurbitacin B may be a potential candidate for the management of cervical cancer by inducing apoptosis, cell cycle arrest and regulating the mTOR/PI3K/Akt signaling pathway. There are limited effective treatments available for cervical cancer; the low toxicity associated with the naturally occurring 23,24-dihydrocucurbitacin B means that it may be developed as a novel treatment for cervical cancer. However, further studies are required to validate its effectiveness in cervical cancer.

\section{References}

1. Chen JC, Chiu MH, Nie RL, Cordell GA and Qiu SX: Cucurbitacins and cucurbitane glycosides: Structures and biological activities. Nat Prod Rep 22: 386-399, 2005.

2. Ishii T, Kira N, Yoshida T and Narahara H: Cucurbitacin D induces growth inhibition, cell cycle arrest, and apoptosis in human endometrial and ovarian cancer cells. Tumour Biol 34: 285-291, 2013.

3. Kapoor S: Cucurbitacin B and its rapidly emerging role in the management of systemic malignancies besides lung carcinomas. Cancer Biother Radiopharm 28: 359, 2013.

4. Lui VW, Yau DM, Wong EY, Ng YK, Lau CP, Ho Y, Chan JP, Hong B, Ho K, Cheung CS, et al: Cucurbitacin I elicits anoikis sensitization, inhibits cellular invasion and in vivo tumor formation ability of nasopharyngeal carcinoma cells. Carcinogenesis 30: 2085-2094, 2009.

5. Jemal A, Bray F, Center MM, Ferlay J, Ward E and Forman D: Global cancer statistics. CA Cancer J Clin 61: 69-90, 2011.

6. Cadron I, Van Gorp T, Amant F, Leunen K, Neven P and Vergote I: Chemotherapy for recurrent cervical cancer. Gynecol Onco 107 (1 Suppl 1): S113-S118, 2007.

7. Engelman JA: Targeting PI3K signalling in cancer: Opportunities, challenges and limitations. Nat Rev Cancer 9: 550-562, 2009

8. Romashkova JA and Makarov SS: NF-kappaB is a target of AKT in anti-apoptotic PDGF signalling. Nature 401: 86-90, 1999.

9. Chiang JH, Yang JS, Ma CY, Yang MD, Huang HY, Hsia TC, Kuo HM, Wu PP, Lee TH and Chung JG: Danthron, an anthraquinone derivative, induces DNA damage and caspase cascades-mediated apoptosis in SNU-1 human gastric cancer cells through mitochondrial permeability transition pores and Bax-triggered pathways. Chem Res Toxicol 24: 20-29, 2011.

10. Sun SY, Hail N Jr and Lotan R: Apoptosis as a novel target for cancer chemoprevention. J Natl Cancer Inst 96: 662-672, 2004.

11. Maitra R, Porter MA, Huang S and Gilmour BP: Inhibition of NFkappaB by the natural product Withaferin A in cellular models of Cystic Fibrosis inflammation. J Inflamm (Lond) 6: 15, 2009.

12. Chiang LC, Ng LT, Lin IC, Kuo PL and Lin CC: Anti-proliferative effect of apigenin and its apoptotic induction in human Hep G2 cells. Cancer Lett 237: 207-214, 2006.

13. Ren S, Ouyang DY, Saltis M, Xu LH, Zha QB, Cai JY and He XH: Anti-proliferative effect of 23,24-dihydrocucurbitacin F on human prostate cancer cells through induction of actin aggregation and cofilin-actin rod formation. Cancer Chemother Pharmacol 70: 415-424, 2012.

14. Yang L, Wu S, Zhang $Q$, Liu $F$ and $W u$ P: 23,24-Dihydrocucurbitacin B induces $\mathrm{G} 2 / \mathrm{M}$ cell-cycle arrest and mitochondria-dependent apoptosis in human breast cancer cells (Bcap37). Cancer Lett 256: 267-278, 2007.

15. Hissin PJ and Hilf R: A fluorometric method for determination of oxidized and reduced glutathione in tissues. Anal Biochem 74: 214-226, 1976. 
16. Chipuk JE, Bouchier-Hayes L and Green DR: Mitochondrial outer membrane permeabilization during apoptosis: The innocent bystander scenario. Cell Death Diff 13: 1396-1402, 2006.

17. Azuma M, Tamatani T, Ashida Y, Takashima R, Harada K and Sato M: Cisplatin induces apoptosis in oral squamous carcinoma cells by the mitochondria-mediated but not the NF-kappaB-suppressed pathway. Oral Oncol 39: 282-289, 2003.

18. Yoneda K, Yamamoto T and Osaki T: p53- and p21-independent apoptosis of squamous cell carcinoma cells induced by 5 -fluorouracil and radiation. Oral Oncol 34: 529-537, 1998.

19. Abal M, Andreu JM and Barasoain I: Taxanes: Microtubule and centrosome targets and cell cycle dependent mechanisms of action. Curr Canc Drug Targs 3: 193-203, 2003.

20. Ferreira CG, Epping M, Kruyt FA and Giaccone G: Apoptosis: Target of cancer therapy. Clin Cancer Res 8: 2024-2034, 2002.

21. Indran IR, Hande MP and Pervaiz S: hTERT overexpression alleviates intracellular ROS production, improves mitochondrial function, and inhibits ROS-mediated apoptosis in cancer cells. Cancer Res 71: 266-276, 2011.

22. Sharma V, Anderson D and Dhawan A: Zinc oxide nanoparticles induce oxidative DNA damage and ROS-triggered mitochondria mediated apoptosis in human liver cells (HepG2). Apoptosis 17 $852-870,2012$
23. Li AX, Sun M and Li X: Withaferin-A induces apoptosis in osteosarcoma U2OS cell line via generation of ROS and disruption of mitochondrial membrane potential. Eur Rev Med Pharmacol Sci 21: 1368-1374, 2017

24. Sun H, Wang Z and Sebastian Yakisich J: Natural products targeting autophagy via the PI3K/Akt/mTOR pathway as anticancer agents. Anticancer Agents Med Chem 13: 1048-1056, 2013.

25. Matsuda S, Ichimura M, Ogino M, Nakano N, Minami A, Murai T and Kitagishi Y: Effective PI3K modulators for improved therapy against malignant tumors and for neuroprotection of brain damage after tumor therapy (Review). Int J Oncol 49: 1785-1790, 2016.

26. Caporali S, Alvino E, Lacal PM, Levati L, Giurato G, Memoli D, Caprini E, Antonini Cappellini GC and D'atri S: Targeting the $\mathrm{PI} 3 \mathrm{~K} / \mathrm{AKT} / \mathrm{mTOR}$ pathway overcomes the stimulating effect of dabrafenib on the invasive behavior of melanoma cells with acquired resistance to the BRAF inhibitor. Int J Oncol 49: 1164-1174, 2016

27. Blair J, Barry R, Moore DJ and Denniston AK: A comprehensive review of mTOR-inhibiting pharmacotherapy for the treatment of non-infectious uveitis. Curr Pharm Des 23: 3005-3014, 2017.

28. Nicoletti F, Fagone P, Meroni P, McCubrey J and Bendtzen K: mTOR as a multifunctional therapeutic target in HIV infection. Drug Discoy Today 16: 715-721,2011. 